\title{
1 Reporter gene assay for membrane fusion of extracellular vesicles
}

2

3 Masaharu Somiya ${ }^{1, *}$, and Shun'ichi Kuroda ${ }^{1}$

$4 \quad{ }^{1}$ The Institute of Scientific and Industrial Research, Osaka University, Osaka 567-0047,

5 Japan

6

$7 \quad$ *Corresponding author: Prof. Masaharu Somiya, Ph.D

8 Department of Biomolecular Science and Reaction, The Institute of Scientific and

9 Industrial Research, Osaka University, 8-1 Mihogaoka, Ibaraki, Osaka 567-0047, Japan

10 E-mail: msomiya@sanken.osaka-u.ac.jp

11 Phone: 81-6-6879-8462

12 


\section{Abstract}

15 Extracellular vesicles (EVs) secreted by living cells are expected to deliver biological

16 cargo molecules, including RNA and proteins, to the cytoplasm of recipient cells. There

17 is an increasing need to understand the mechanism of intercellular cargo delivery by EVs.

18 However, the lack of a feasible bioassay has hampered our understanding of the biological

19 processes of EV uptake, membrane fusion, and cargo delivery to recipient cells. Here, we

20 describe a reporter gene assay that can measure the membrane fusion efficiency of EVs

21 during cargo delivery to recipient cells. When EVs containing tetracycline transactivator

22 (tTA)-fused tetraspanins are internalized by recipient cells and fuse with cell membranes,

23 the tTA domain is exposed to the cytoplasm and cleaved by protease to induce

24 tetracycline responsive element (TRE)-mediated reporter gene expression in recipient

25 cells. This assay (designated as EV-mediated tetraspanin-tTA delivery assay, ETTD

26 assay), enabled us to assess the cytoplasmic cargo delivery efficiency of EVs in recipient

27 cells. With the help of a vesicular stomatitis virus-derived membrane fusion protein, the

28 ETTD assay could detect significant enhancement of cargo delivery efficiency of EVs.

29 Furthermore, the ETTD assay could evaluate the effect of potential cargo delivery

30 enhancers/inhibitors. Thus, the ETTD assay may contribute to a better understanding of

31 the underlying mechanism of the cytoplasmic cargo delivery by EVs. 
bioRxiv preprint doi: https://doi.org/10.1101/2021.02.16.431359; this version posted February 17, 2021. The copyright holder for this preprint (which was not certified by peer review) is the author/funder. All rights reserved. No reuse allowed without permission.

33 Keywords: cargo transfer; extracellular vesicles; membrane fusion; NanoLuc; VSV-G

34 


\section{Introduction}

36 Extracellular vesicles (EVs) are secreted by living cells and contain biomolecules derived

37 from the donor cells. The physiological role of EVs remains largely unknown and they

38 were formerly known as the "garbage bin" of cells for excretion of the unwanted

39 molecules or organelles. Several studies have shown the cellular disposal role of EVs ${ }^{1,2}$

40 although a vast majority of current EV research focuses on the cargo delivery of EVs.

41 Since EVs contain cargo proteins and RNAs, their contents can be transferred from a

42 donor cell to a recipient cell via a paracrine or endocrine mechanism. Recently, EV-

43 mediated cargo delivery events in pathophysiological settings, such as cancers, have

44 attracted considerable attention. Several studies have reported that EVs are involved in

45 tumor suppression ${ }^{3,4}$ and tumor progression ${ }^{5,6}$. Several studies have demonstrated that

46 EVs can deliver small RNAs to recipient cells and elicit phenotypic changes. However,

47 there is limited evidence that demonstrates cargo delivery by EVs into recipient cells ${ }^{7}$.

48 Many confounding factors in the experimental conditions and contaminants in the EV

49 fraction ${ }^{8}$ must be taken into account in the cargo delivery experiments, to draw a

50 conclusion on "EV cargo transfer hypothesis" 9. 
53 reporter assays that demonstrate the functional delivery of cargo proteins or RNAs have

54 been reported, including miRNA ${ }^{11,12}$, Cre-LoxP ${ }^{13,14}$, and CRISPR/Cas9-gRNA reporters

$55 \quad 15$. However, these assays are influenced by various confounding factors including non-

56 EV components in the EV fraction. Although the readout of these assays is informative

57 for deciphering the delivery mechanism of EVs in recipient cells, a more precise reporter

58 assay is needed. Mechanistically, cytoplasmic cargo delivery should occur after

59 endocytosis and subsequent membrane fusion, or direct fusion with the plasma membrane

$60{ }^{16}$. Upon membrane fusion, the luminal side of EVs is exposed to the cytoplasm of

61 recipient cells and release their cargo. The functional delivery assay should reflect the

62 biological delivery mechanism, especially membrane fusion of EVs.

In this study, we developed a reporter assay to quantify the membrane fusion of

64 EVs in recipient cells. In this assay, following fusion of EVs with the cell membrane of

65 the recipient cells, a transcription factor is released from the EVs and then upregulates

66 the expression of a reporter gene (luciferase or fluorescence protein). This assay provides

67 a biologically orthogonal readout and enables us to accurately interpret the cargo delivery

68 process of EVs.

69 


\section{$71 \quad$ Materials and Methods}

\section{$72 \quad$ Materials}

73 The chemical reagents and antibodies used in this study are listed in Supplementary Table

74 1. All NanoLuc substrates were purchased from Promega. The plasmids used in this study

75 are listed in Supplementary Table 2 and deposited at Addgene. Plasmids were constructed

76 using PCR-based methods (Gibson Assembly ${ }^{17}$ ) and confirmed by Sanger sequencing.

\section{Cell culture and transfection}

79 Human embryonic kidney HEK293T cells (RIKEN Cell Bank) were maintained in 10\%

80 (v/v) fetal bovine serum (FBS)-containing Dulbecco's modified Eagle's medium

81 (DMEM) supplemented with $10 \mu \mathrm{g} / \mathrm{mL}$ penicillin-streptomycin solution. Cells were cultured at $37^{\circ} \mathrm{C}$ under $5 \% \mathrm{CO}_{2}$ in humidified conditions. Transfection of HEK293T cells was performed as follows: cells were plated in a

84 cell culture dish or multi-well plate and cultured overnight. The next day, the cells were

85 transfected using 25-kDa branched polyethyleneimine (PEI, Sigma). The ratio of plasmid

86 DNA to PEI was 1: 4 (weight). After 24-96 h, the cells were used in the subsequent

87 experiments. Cell culture supernatant was collected after 2-4 days and centrifuged at $1,500 \times \mathrm{g}$ for $5 \mathrm{~min}$ to remove cell debris. 
$90 \quad$ NanoLuc assay

91 To quantify the expression level of the reporter NanoLuc, the transfected cells were lysed

92 and mixed with NanoLuc substrate (Nano-Glo Luciferase Assay System; Promega)

93 according to the manufacturer's instructions. Luminescence signal from the cell lysate

94 was measured by using a plate reader, Synergy 2 (BioTek).

95

96 Characterization of tTA-fused proteins in cell lysate and EVS

97 Protein expression was assessed by western blotting. Briefly, lysates of the transfected

98 cells (total protein was extracted using radioimmunoprecipitation assay [RIPA] buffer

99 [Nacalai Tesque] containing a protease inhibitor cocktail [Nacalai Tesque]) or the

100 supernatant was mixed with reductant-free sample buffer and incubated at room

101 temperature for $20 \mathrm{~min}$. Proteins were separated by sodium dodecyl sulfate

102 polyacrylamide gel electrophoresis (SDS-PAGE) and transferred to polyvinylidene

103 difluoride (PVDF) membrane. Proteins on the membrane were detected using antibodies

104 (Supplementary Table 1) and ImmunoStar LD reagent (FUJIFILM Wako Pure Chemical).

105 As a loading control for cell lysates, the membrane was probed with anti-GAPDH

106 antibody. 
109 EVs were concentrated by PEG precipitation. The supernatant was mixed with 4× PEG

110 solution (40\% PEG 6000 [w/v], $1.2 \mathrm{M} \mathrm{NaCl}, 1 \times \mathrm{PBS}[\mathrm{pH} 7.4])$, and kept at $4^{\circ} \mathrm{C}$ overnight.

111 The next day, the supernatant was centrifuged at $1,600 \times \mathrm{g}$ for $60 \mathrm{~min}$ to pellet the EVs.

112 After decantation, the pellet was resuspended in PBS. Typically, 5-10 mL of the

113 supernatant was concentrated to $100-200 \mu \mathrm{L}$.

115 Reporter assay

116 For the membrane fusion reporter assay, recipient HEK293T cells $\left(10^{4}\right.$ cells/well in $96-$

117 well plate) were transfected with plasmids encoding tobacco etch virus (TEV) protease

118 (TEVp) and TRE3G-NlucP (PEST motif-fused NanoLuc $[\text { NlucP }]^{18}$ under tetracycline

119 responsive element [TRE] promoter), and cultured overnight. The next day, the recipient

120 cells were treated with donor culture supernatant or concentrated EVs and further

121 incubated at $37^{\circ} \mathrm{C}$ for up to $26 \mathrm{~h}$. To assess the effect of various compounds on membrane

122 fusion efficiency, recipient cells were treated with the compound $1 \mathrm{~h}$ before the addition

123 of supernatant or EVs. After incubation (2-26 h), the expression of NanoLuc in the

124 recipient cells was measured as described above. 
126 green fluorescent protein (EGFP) gene. Recipient cells $\left(10^{4}\right.$ cells/well in 96 -well plate)

127 transfected with pTetOn-EGFP (EGFP under TRE promoter) and pcDNA3.1-TEVp were

128 treated with EVs, and then observed under a fluorescence microscope IX70 (Olympus)

129 after $24 \mathrm{~h}$. Cre recombinase-based reporter assay was performed in the same way;

130 recipient HEK293T cells were transfected with reporter plasmid (encoding LoxP-flanked

131 mKate and EGFP under the CMV promoter) and plasmid encoding TEVp, treated with

132 EVs for $24 \mathrm{~h}$ the following day, and then observed under a fluorescence microscope.

134 Statistical analysis

135 Data were analyzed using Student's $t$-test or one-way ANOVA following either post hoc

136 Tukey's HSD or Dunnett's tests. Statistical analysis was performed using the Real

137 Statistics Resource Pack software created by Charles Zaiontz. 


\section{Results}

139 Characterization of tTA-fused tetraspanins

140 To establish a reporter assay that can measure the membrane fusion of EVs, we first

141 prepared plasmids encoding human tetraspanins CD9, CD63, or CD81 with C-terminal

142 fusion of the TEVp cleavage site, followed by tTA (Fig. 1A). As shown in Fig. 1B, tTA-

143 fused tetraspanin is cleaved in the presence of TEVp and releases the transcription

144 activator tTA. When the EVs containing tTA-fused tetraspanin are internalized and fused

145 with the endosomal membrane, luminal tTA are exposed to the cytoplasmic side, and

146 TEVp in the recipient cells cleaves the TEVp site, followed by cytoplasmic release of

147 tTA and induction of the reporter gene expression under the TRE promoter (Fig. 1C). We

148 designated this assay the EV-mediated tetraspanin-tTA delivery (ETTD) assay. 

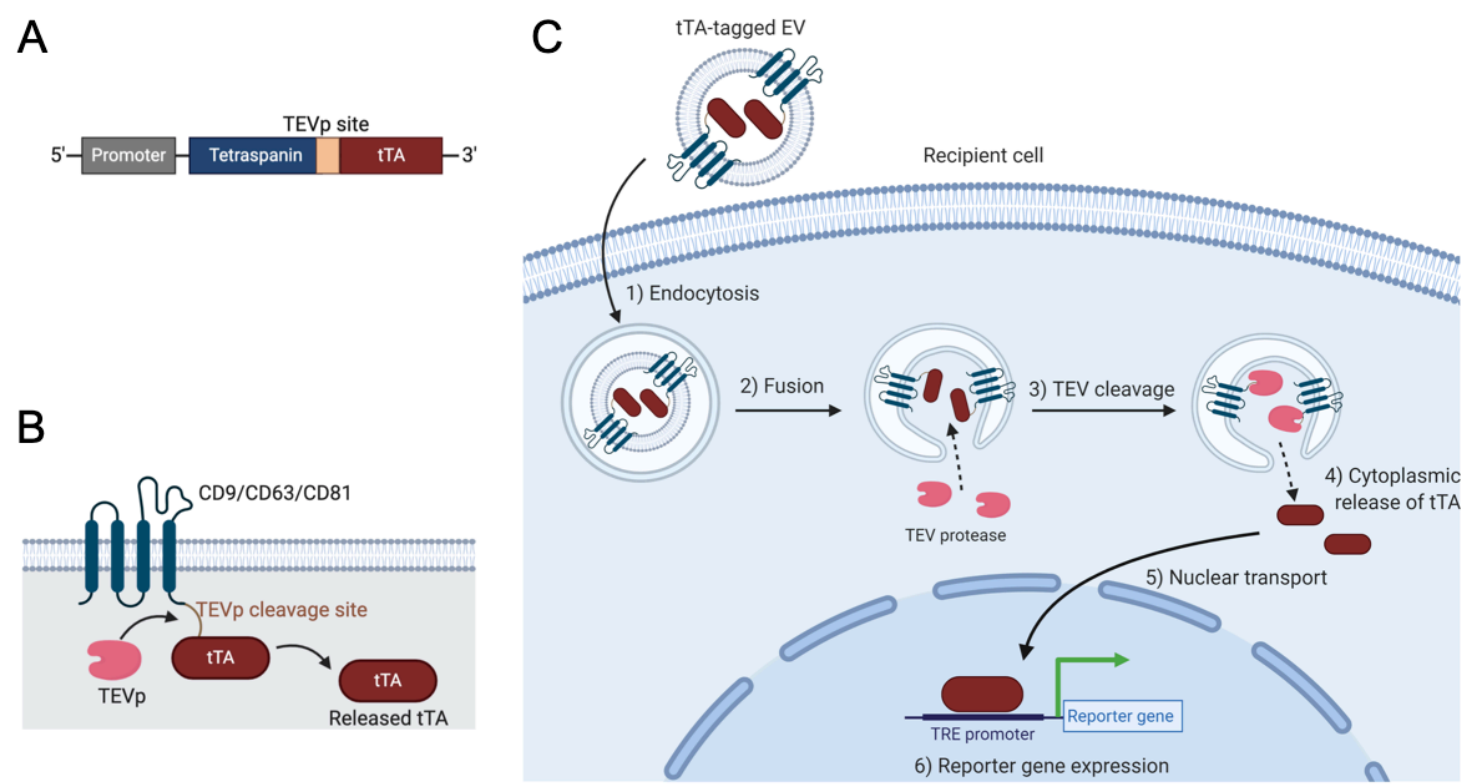

B

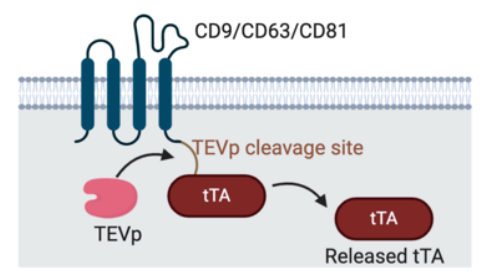

151 Fig. 1 Summary of the ETTD assay.

152 (A) Schematic representation of tTA-fused tetraspanin. Tetraspanin and tTA flank a

153 TEVp recognition site.

154 (B) Topology of tTA-fused tetraspanin protein. Upon the cleavage by TEVp, tTA is

155 released from membrane-anchored tetraspanin.

156 (C) Schematic representation of the ETTD assay. EV containing tTA-fused tetraspanin is

157 taken up by cells by endocytosis (1), and fuses with the endosomal membrane (2). After

158 cleavage by cytoplasmic TEVp (3), tTAs are released into the cytoplasm (4). Released

159 tTAs are transported to nucleus (5), and induce expression of reporter gene under TRE promoter (6). 
164 encoding TEVp. As shown in Fig. 2A, the expression of tTA-tetraspanins in the cell lysate

165 was confirmed by western blotting. In the presence of TEVp, tTA was cleaved and

166 released from the tTA-fused protein. While CD9 and CD81 showed obvious tTA bands,

167 CD63 showed only a weak band in the absence of TEVp and no band in the presence of

168 TEVp. This is probably due to low expression of CD63 in HEK293T cells compared to

169 CD9 and CD81. When HEK293T cells were transfected with both NlucP (under the TRE

170 promoter) and tTA-fused proteins, co-expression of TEVp strongly induced Nluc

171 expression (Fig. 2B), suggesting that tetraspanin-anchored tTA was unable to translocate

172 into the nucleus, and therefore could not induce reporter gene expression. In contrast,

173 expression of non-fused tTA protein continually induced reporter gene expression

174 regardless of the co-expression of TEVp. These results suggest that tTA-fused

175 tetraspanins induce reporter gene expression in the recipient cells only when the cells 
A

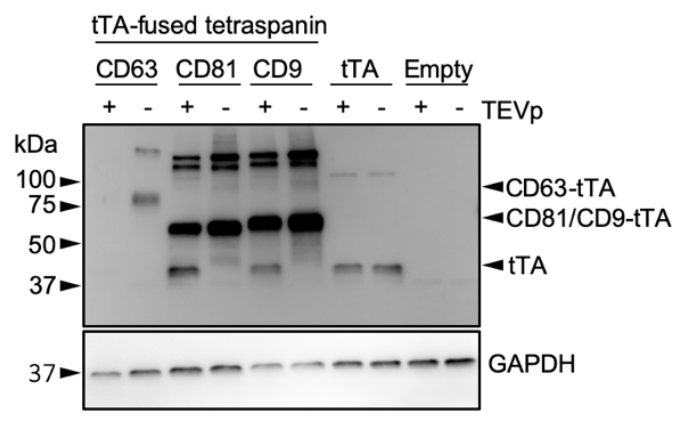

B

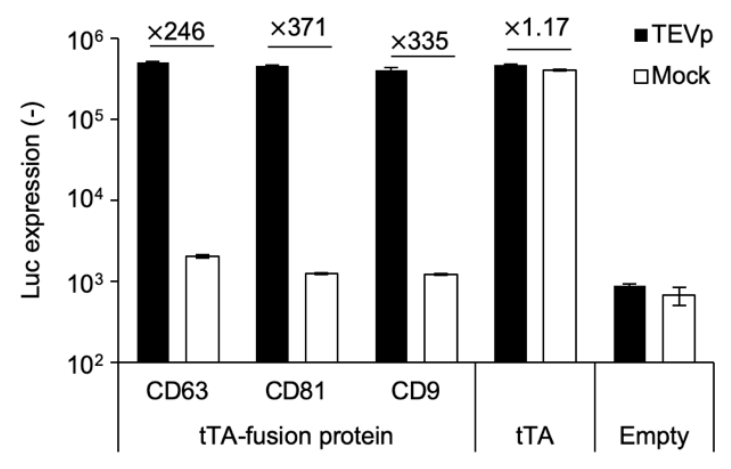

177 Fig. 2 Characterization of tTA-fused tetraspanins.

178 (A) Cells were transfected with plasmids encoding tTA-fused tetraspanins and TEVp.

179 After $48 \mathrm{~h}$, cells were lysed and subjected to western blotting. Upper and lower panels

180 represent immunoblotting using anti-TetR antibody and anti-GAPDH antibody,

181 respectively. The expected molecular weights based on the amino acid sequences were as

182 follows: CD63-tTA, 63.2 kDa; CD81-tTA, 63.4 kDa; CD9-tTA, 63.0 kDa; tTA, 36.9 kDa.

183 (B) Expression of NanoLuc under TRE3G promoter in HEK293T cells co-expressing

184 tTA-fused tetraspanins and TEVp. As controls, plasmids encoding tTA without

185 tetraspanin fusion and empty expression plasmid were used. Numbers above the bars

186 indicate the fold increase in NanoLuc expression compared to the mock transfection. $\mathrm{N}=$ 

and analyzed by western blotting (Fig. 3A and 3B). Both tTA-fused CD81 and CD9 were detected with anti-CD81 and CD9 antibodies, respectively. The tTA-fused proteins were also detected with an anti-TetR antibody, indicating that the released EVs contain full-

194 length tTA-fused CD81 or CD9. As a control for the ETTD assay, vesicular stomatitis virus glycoprotein (VSV-G) was co-expressed in donor cells, as VSV-G is known to

A

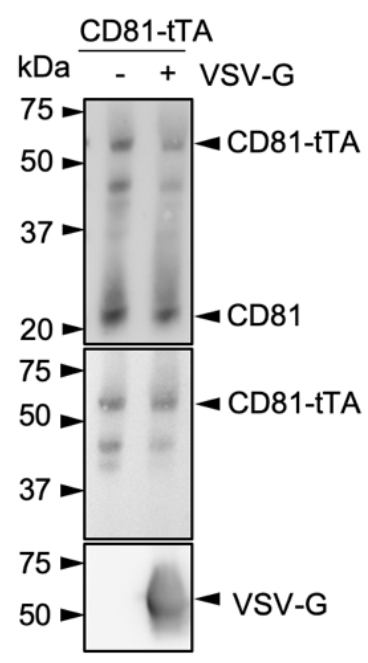

B

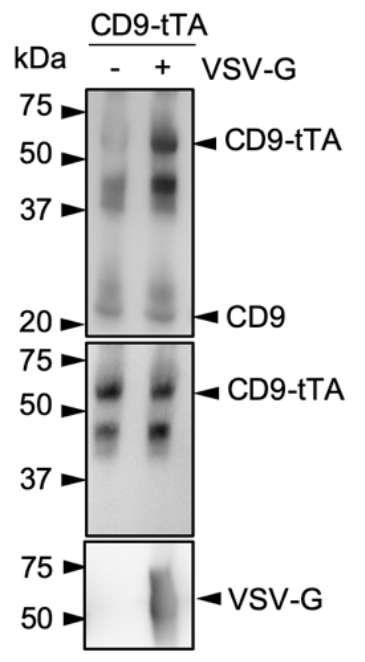

Fig. 3 Characterization of HEK293Tderived EVs containing tTA-fused tetraspanins by western blotting.

(A) EVs containing CD81-tTA with or without VSV-G. Antibodies used were as follows; top, anti-CD81 antibody; middle, 
anti-TetR antibody; bottom, anti-VSV-G antibody.

207 (B) EVs containing CD9-tTA with or without VSV-G. Antibodies used were as follows;

208 top, anti-CD9 antibody; middle, anti-TetR antibody; bottom, anti-VSV-G antibody.

209 The expected molecular weights based on the amino acid sequences were as follows:

210 CD81-tTA, 63.4 kDa; CD9-tTA, 63.0 kDa; VSV-G, 57.7 kDa.

212 Validation of ETTD assay for cargo delivery of EVS

213 We first attempted to assess whether the unconcentrated cell culture supernatant from

214 donor cells was capable of inducing reporter gene expression in recipient cells. As shown

215 in Fig. 4A, treatment of recipient cells with donor supernatant containing tetraspanin-tTA

216 fusion protein induced reporter gene expression only when the donor cells were

217 transfected with virus-derived fusogenic protein VSV-G. This result suggested that the

218 concentration process is not necessary to evaluate EV membrane fusion in the ETTD

219 assay if the EVs possessed potent fusogenic activity. While the supernatant containing

220 tTA-fused CD81 and CD9 induced > 10-fold increase in NanoLuc expression, the

221 supernatant containing tTA-fused CD63 showed less induction (up to 5-fold). This may

222 reflect the lower expression level of tTA-fused CD63 in the donor cells compared to CD9 
A

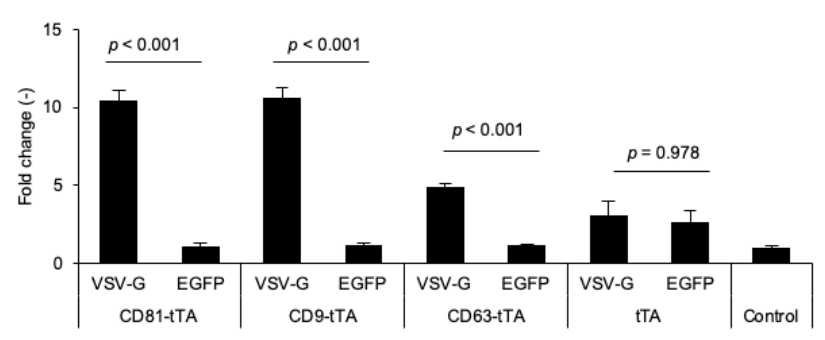

$\mathrm{C}$
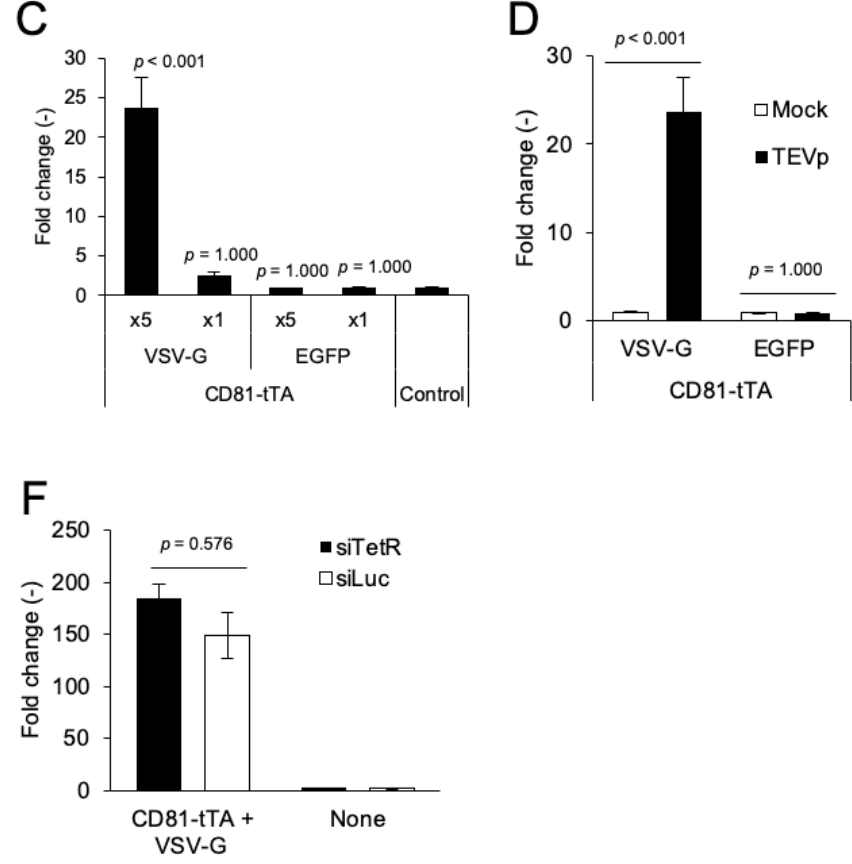

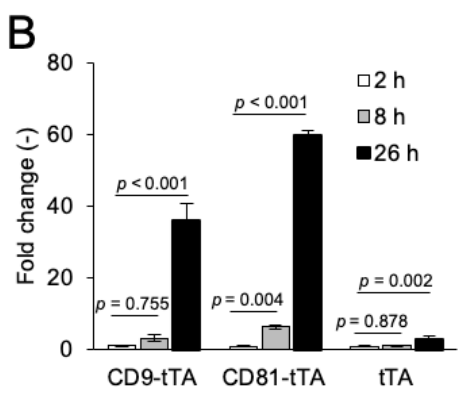

$\mathrm{E}$

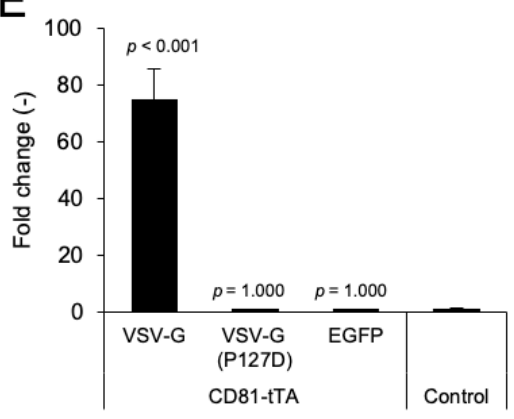

225 Fig. 4 ETTD assay.

226 (A) Donor supernatant was applied to recipient HEK293T cells and NanoLuc expression

227 was measured after $24 \mathrm{~h}$. NanoLuc expression was normalized to the control (treatment

228 with supernatant from non-transfected donor cells). 
229 (B) Incubation time-dependent expression of reporter gene. Recipient cells were treated

230 with concentrated VSV-G-expressing EVs containing tTA-fused CD9 or CD81 for 2, 8,

231 and $26 \mathrm{~h}$, followed by luciferase assay.

232 (C) Dose-dependent reporter expression in recipient cells. Recipient cells were treated

233 with EVs containing tTA-fused CD81 with or without VSV-G for $24 \mathrm{~h}$. The relative

234 amount of EV fraction added was noted as $\times 1$ or $\times 5$.

235 (D) TEVp-dependent reporter gene expression. Recipient cells with or without expression

236 of TEVp were treated with EVs containing tTA-fused CD81 with or without VSV-G and

237 subjected to the luciferase assay after $24 \mathrm{~h}$.

238 (E) Effect of fusogenicity deficit VSV-G mutant. Recipient cells were treated with EVs

239 (tTA-fused CD81) displaying parental VSV-G, mutant VSV-G (P127D), or EGFP for 24

$240 \mathrm{~h}$

241 (F) Recipient cells were pre-transfected with siRNAs targeting TetR (siTetR) or firefly

242 luciferase (siLuc), and further treated with EVs containing tTA-fused CD81 and VSV-G

243 for $24 \mathrm{~h}$. 
$244 \mathrm{~N}=3$, mean $\pm \mathrm{SD}$. Statistical analysis was performed using one-way ANOVA followed

245 by post hoc Tukey's HSD (A, B, D, E, and F) or Dunnett's tests (C).

248 Recipient cells were treated with EVs for 2, 8, and $26 \mathrm{~h}$ and the reporter NanoLuc

249 expression was measured (Fig. 4B). NanoLuc expression gradually increased over time

250 and reached a highest level at $26 \mathrm{~h}$. The expression of NanoLuc was detected as early as

$2518 \mathrm{~h}$. Induction of NanoLuc expression was dependent on the presence of VSV-G and the

252 dose of EVs (Fig. 4C). Fig. 4D indicates that expression of TEVp in the recipient cells

was crucial for reporter gene expression, demonstrating that the ETTD assay worked as

254 expected (Fig. 1C). Furthermore, the EVs harboring fusion-deficient mutants of VSV-G

255 (P127D) ${ }^{19,22}$ lost the membrane fusion ability of EVs in the assay compared to the EVs

256 harboring parent VSV-G (Fig. 4E), strongly supporting that this assay depicted the

257 membrane fusion-mediated cargo delivery of EVs. Furthermore, absence of VSV-G led

258 to no functional delivery (Fig. 4C to 4E), indicating the poor cargo delivery efficacy of

259 authentic EVs. In addition to HEK293T cells, HeLa cells were used as alternative

260 recipient cells, and similar results were observed, indicating that the ETTD assay is

261 potentially applicable to other cell lines (Fig. S1). 
263 supernatant or mRNA of tTA-fused tetraspanin encapsulated in EVs may induce the

264 reporter gene expression in the recipient cells, which could confound the bona fide

265 reporter expression due to the tTA release of EVs. Therefore, we transfected the reporter

266 cells with siRNA targeting TetR, the TRE-binding domain of tTA to verify that the

267 reporter gene expression was induced by tTA protein. First, we verified that siRNA

268 targeting TetR (siTetR) efficiently knocked down tTA (Fig. S2A). Furthermore,

269 knockdown of tTA by siRNA significantly suppressed TRE-mediated reporter gene

270 expression (Fig. S2B). Based on these results, siRNA targeting tTA should abrogate the

271 confounding factors in the ETTD assay, namely, the excess of expression plasmid

272 remaining in the donor supernatant and mRNA-mediated expression of tTA. After

273 transfection of siRNA into recipient cells, we applied tTA-fused EVs to recipient cells.

274 As shown in Fig. 4F, transfection of siRNA targeting tTA showed no effect on the reporter

275 gene expression, strongly suggesting that the assay readout of the ETTD assay was solely

276 driven by tTA proteins, neither mRNA nor leftover plasmid DNA. 
279 As the novel ETTD assay can evaluate the membrane fusion efficiency of EVs, we next

280 examined the effect of potential delivery enhancers and entry inhibitors. According to a

281 previous report, chloroquine enhanced Cre protein delivery of EVs by disrupting

282 endosomes and lysosomes using the Cre-LoxP reporter assay ${ }^{14}$. In our reporter assay,

283 chloroquine treatment did not induce any reporter gene expression (Fig. 5A), suggesting

284 that chloroquine does not enhance cytoplasmic cargo delivery of EVs. This is probably

285 because chloroquine treatment induces the destabilization of endosomes/lysosomes and

286 does not enhance membrane fusion. 


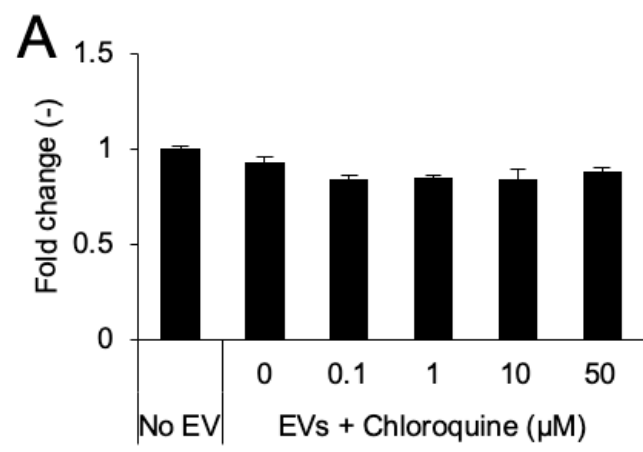

B
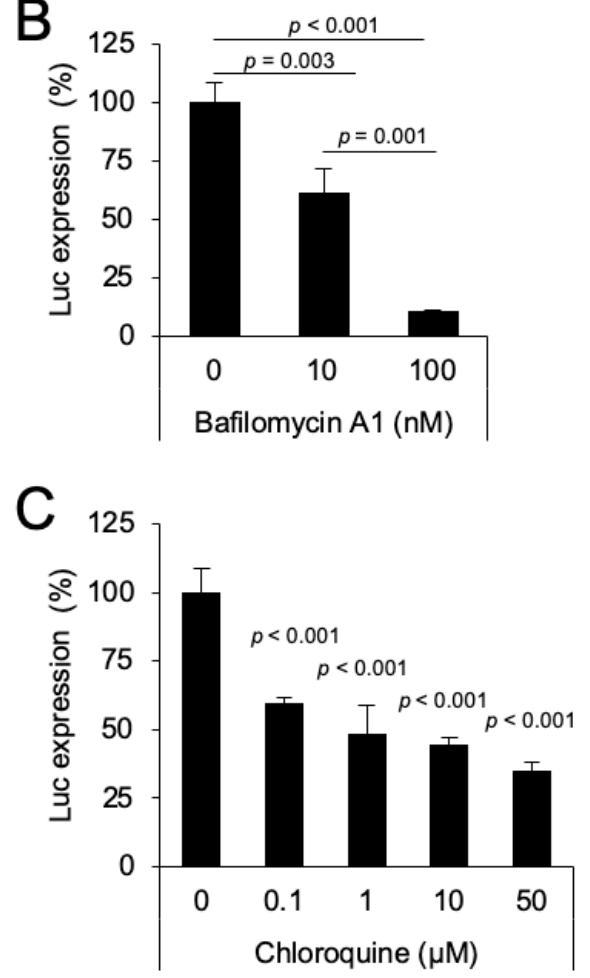

Fig. 5 Effect of small molecule compounds on the ETTD assay.

(A) EVs (CD81-tTA) without VSV-G were applied to recipient HEK293T cells in the presence of indicated concentrations of chloroquine. NanoLuc expression level was normalized to the value of the control (no EV treatment) and is presented as fold-change. (B) EVs (CD81-tTA) with VSV-G were applied to recipient cells in the presence of 10 or $100 \mathrm{nM}$ of bafilomycin A1.

(C) EVs (CD81-tTA) with VSV-G were

301 applied to recipient cells in the presence of 0.1 to $50 \mu \mathrm{M}$ of chloroquine. by post hoc Tukey's HSD test. 
307 compounds that are known to increase the endosomal $\mathrm{pH}$ and thereby inhibit the low $\mathrm{pH}-$

308 dependent fusion activity of VSV-G ${ }^{23,24}$. Bafilomycin A1 is a selective ATPase inhibitor

30925 that prevents the acidification of endosomes/lysosomes and inhibits VSV infection ${ }^{26}$.

310 When recipient cells were treated with bafilomycin A1, membrane fusion by VSV-G-

311 modified EVs was significantly inhibited in a dose-dependent manner (Fig. 5B). In

312 addition, chloroquine, which is known to prevent VSV infection by increasing

313 endosomal/lysosomal $\mathrm{pH}^{27}$, also blocked the membrane fusion of EVs (Fig. 5C). These

314 results strongly support the application of ETTD assay in assessing pharmacological

315 effects of a potential delivery enhancer/inhibitor of EVs.

317 Assessment of membrane fusion efficiency of EVs at the single-cell level

318 For the evaluation of EV membrane fusion at the single-cell level, we changed the

319 reporter gene from NanoLuc to EGFP. As shown in Fig. 6A, EVs containing tTA-fused

320 CD81 and VSV-G induced EGFP expression in the recipient cells, which was consistent

321 with previous results (Fig. 4). This assay enabled us to decipher membrane fusion

322 efficiency at the single-cell level. 
325 dependent on Cre recombinase. The principle of the Cre-mediated reporter assay is

326 essentially the same as that of the ETTD assay; however, the readout is driven by Cre-

327 mediated recombination of the target gene. After the release of Cre from EV by TEVp,

328 Cre recombinases translocate to the nucleus and induce recombination of the target

329 plasmid (Fig. 6B \& 6C). In this study, we used the mKate/EGFP reporter plasmid. The

330 recipient cells initially expressed the red fluorescence protein mKate, but after Cre-

331 mediated recombination, cells become EGFP positive (Fig. 6C). This assay may be more

332 sensitive than the tTA reporter assay as even a single molecule of Cre recombinase can

333 induce a readout in the recipient cells. As shown in Fig. 6D, EVs containing CD81-Cre

334 with VSV-G induced EGFP positive cells, whereas EVs with VSV-G (P127D) showed

335 almost no EGFP positive cells. This result was consistent with the results of the previous

336 ETTD assay (Fig. 4) and again revealed that fusogenic proteins significantly increase the

337 membrane fusion activity of EVs. 
A

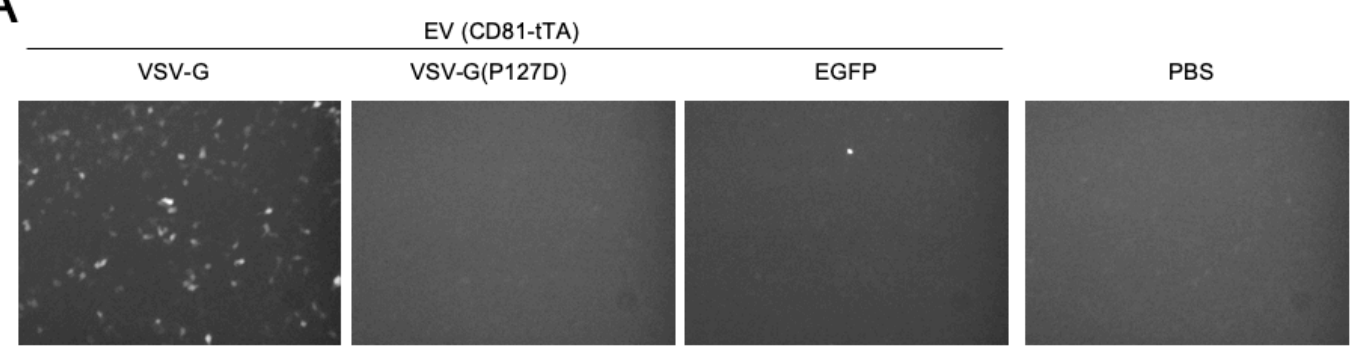

B

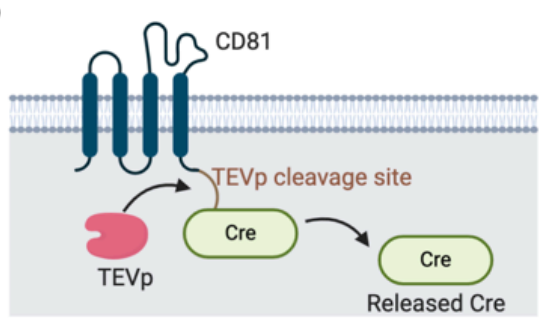

C

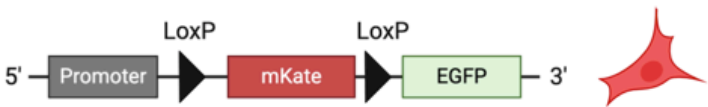

D
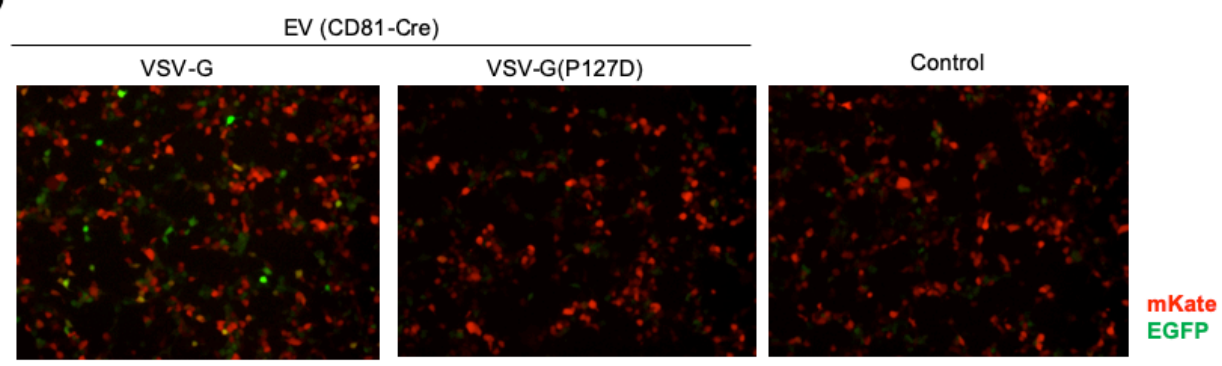

$340 \quad$ Fig. 6 Fluorescence imaging-based reporter assays

341 (A) EDDT assay based on EGFP as a reporter readout. Recipient HEK293T cells were

342 transfected with plasmids encoding TRE3G-EGFP and TEVp, and treated with EVs

343 containing CD81-tTA with VSV-G (WT or P127D mutant) or EGFP (negative control).

344 After $24 \mathrm{~h}$, cells were observed under a fluorescence microscope.

(B) Schematic representation of Cre-fused CD81. 
346 (C) Schematic representation of reporter plasmid for Cre-LoxP reporter assay. After the

347 Cre cleavage, mKate gene is excised and reporter cells become EGFP positive.

348 (D) Fluorescence imaging of Cre-LoxP reporter assay. Recipient cells were treated with

349 EVs harboring CD81-Cre and VSV-G.

350 


\section{Discussion}

352 In this study, we developed an ETTD assay that can evaluate the membrane fusion

353 efficiency of EVs in recipient cells. The principle of this assay was inspired by the tango

354 assay ${ }^{28}$ that quantitatively assesses receptor activation by the recruitment of genetically

355 engineered TEVp to the receptor, subsequent release of tTA, and expression of TRE-

356 mediated reporter gene. In the ETTD assay, tetraspanins constrain tTA and are localized

357 at the membrane (Fig. 2B). Once the luminal tTA is exposed to the cytoplasm following

358 membrane fusion of the EVs and release by TEVp cleavage, recipient cells express the

359 reporter gene (Fig. 4D). This experimental design has rendered the ETTD assay robust

360 and sensitive by avoiding non-specific background signals.

The ETTD assay enables us to quantitatively assess membrane fusion efficiency

and delivery mechanism of EVs. The advantages of this assay are as follows: (1) it is

highly sensitive to measure the membrane fusion of EVs with a wide dynamic range

compared to conventional assays because expression of the reporter gene under the TRE

promoter is highly regulated and specific to the transcription factor tTA, which does not 
368 in both the bulk cell population (NanoLuc reporter) and single-cell level (fluorescence

369 protein reporter).

371 membrane fusion events in recipient cells. Because the cargo delivery efficiency of EVs

372 is expected to be low (possibly $0.2 \%$ to $10 \%$ of recipient cells express reporter gene as a

373 result of the cargo delivery, depending on the reporter assay ${ }^{15,29}$ ), the assay sensitivity

374 must be high to capture the membrane fusion events in recipient cells. When the EVs

375 harbor the fusogenic protein, VSV-G, EV-mediated membrane fusion was sufficient for

376 detection in the ETTD reporter assay, whereas no detectable membrane fusion was

377 observed in the absence of VSV-G (Fig. 4). This result reflected the low efficiency of

378 membrane fusion in the absence of a particular membrane fusion protein. As described in

379 previous studies, the cargo delivery efficiency of EVs is expected to be low $11,15,21,30-32$.

380 However, our experiments were conducted using a combination of HEK293T donor cells

381 and HEK293T or HeLa recipient cells. Other combinations of EV-donor cells and

382 recipient cells should be examined to determine whether the cargo delivery efficiency is

much higher in a future study. 
386 encapsulate overexpressed mRNA in the donor cells in a passive manner and potentially

387 transfer the mRNA to recipient cells ${ }^{33}$. Since it was postulated that unexpected EV-

388 mediated transfer of tTA mRNA may lead to a false positive signal in the ETTD assay,

389 recipient cells were pre-transfected with potent TetR-targeting siRNA (Fig. S2) and

390 blocked the mRNA-mediated readout. The results clearly demonstrated that siRNA

391 targeting TetR did not affect the assay readout, indicating the absence of mRNA-

392 dependent tTA expression and subsequent reporter gene expression in the recipient cells

393 (Fig. 4F).

Previously, membrane fusion of EVs has been evaluated by fluorescence probes

34 or reporter proteins ${ }^{19,20}$. The former approach, especially the membrane-anchored

396 fluorescence probes, such as R18, are known to often result in false positives due to non-

397 specific dye transfer between lipid membranes ${ }^{35}$. Joshi et al. developed a sophisticated

398 fluorescence imaging technique to measure membrane fusion and cargo release of EVs

399 in recipient cells ${ }^{36}$. Their approach enabled the assessment of membrane fusion at the

400 single-vesicle level; however, it was still difficult to distinguish the membrane fusion

401 signal from the high background signal of the fluobodies distributed throughout the

402 cytoplasm, and there was a limited capability in terms of throughput. The latter approach,

403 typically using $\beta$-lactamase (BlaM) protein, is a time-consuming assay that requires a 
404 long incubation time for the enzymatic conversion of a fluorescence substrate ( 7 to $16 \mathrm{~h}$

$40519,20,37$ ). The ETTD assay, in contrast, is more feasible, sensitive, and rapid to assess the

406 membrane fusion process of EVs in recipient cells and capable the high-throughput

407 applications.

408

There are conflicting reports on the effect of chloroquine on EV cargo delivery

409 in a previous study ${ }^{14}$. In this study, chloroquine was unable to enhance membrane fusion

410 and cargo delivery of EVs (Fig. 5A), whereas a previous study showed significant

411 improvement in the Cre delivery of EVs. The inconsistency is probably due to the

412 differences in the experimental settings, sensitivity, and accuracy between assays. The

413 Cre-LoxP reporter assay is a sensitive and robust method since the assay readout is driven

414 by ideally a single Cre molecule in the recipient cell, and assay readout is exclusively

415 dependent on the Cre-LoxP excision of target DNA. The different conclusions between

416 these studies should be carefully interpreted and further examined in a future study. Heath

417 et al. demonstrated that small amounts of Cre recombinase (8.9 Cre-FRB molecules per

418 EV on average) can be passively loaded into EVs and contribute to the recombination in

419 the recipient cells ${ }^{14}$, whereas our approach involved fusion of the Cre protein to the

420 tetraspanin CD81 and application to the reporter recipient cells (Fig. 6B). It appears that

421 our approach may be more convincing because the direct fusion of Cre with the EV 
bioRxiv preprint doi: https://doi.org/10.1101/2021.02.16.431359; this version posted February 17, 2021. The copyright holder for this preprint (which was not certified by peer review) is the author/funder. All rights reserved. No reuse allowed without permission.

422 marker protein is more reliable and precisely reflects the nature of EV-mediated cargo

423 transfer. 


\section{Conclusions}

426 membrane fusion and cargo delivery in a quantitative manner. The lack of reliable

427 functional assays in the EV field has hampered progress in its therapeutic applications ${ }^{38}$

428 and elucidation of the underlying mechanism of cargo delivery and intercellular

429 communication of EVs ${ }^{10}$. The ETTD assay is potentially useful for identifying unknown

430 factors that are responsible for the cargo delivery mechanism. Using the ETTD assay,

431 knockout or knockdown of target genes may reveal the unknown cargo delivery pathway

432 as described in a previous study ${ }^{15}$, or possibly facilitate the discovery of a methodology

433 that enhances membrane fusion and subsequent cargo delivery of EVs. Together with the

434 previously reported real-time cargo delivery assay ${ }^{21}$, the ETTD assay may help advance

435 fundamental EV research and its clinical applications. 


\section{Acknowledgments}

439 We extend our gratitude to Yumi Yukawa for technical assistance in plasmid construction.

440 All illustrations in this work were created using BioRender.com.

441 This work was supported in part by JSPS KAKENHI (Grant-in-Aid for Early-

442 Career Scientists $18 \mathrm{~K} 18386$ and $20 \mathrm{~K} 15790$ to MS), Research Grant from JGC-

443 Scholarship (to MS), and the "Dynamic Alliance for Open Innovation Bridging Human,

444 Environment and Materials" (MEXT).

445 


\section{References}

447 1. Nicolás-Ávila, J. A. et al. A Network of Macrophages Supports Mitochondrial

448 Homeostasis in the Heart. Cell 183, 94-109.e23 (2020).

449 2. Takahashi, A. et al. Exosomes maintain cellular homeostasis by excreting harmful DNA from cells. Nat. Commun. 8, 15287 (2017).

451 3. Seo, N. et al. Activated CD8+ T cell extracellular vesicles prevent tumour progression by targeting of lesional mesenchymal cells. Nat. Commun. 9, (2018).

4. Putz, U. et al. The Tumor Suppressor PTEN Is Exported in Exosomes and Has Phosphatase Activity in Recipient Cells. Sci. Signal. 5, ra70-ra70 (2012). Progression. Cancer Res. 72, 4920-4930 (2012).

6. Ye, S. et al. Tumor-derived exosomes promote tumor progression and T-cell dysfunction through the regulation of enriched exosomal microRNAs in human nasopharyngeal carcinoma. Oncotarget 5, 5439-5452 (2014). extracellular vesicle RNA - An ISEV position paper. J. Extracell. Vesicles 6, 
464 8. Whittaker, T. E., Nagelkerke, A., Nele, V., Kauscher, U. \& Stevens, M. M.

465 Experimental artefacts can lead to misattribution of bioactivity from soluble mesenchymal stem cell paracrine factors to extracellular vesicles. J. Extracell. Vesicles 9, $1807674(2020)$.

9. Somiya, M. Where does the cargo go?: Solutions to provide experimental support for the "extracellular vesicle cargo transfer hypothesis". J. Cell Commun. Signal. 14, 135-146(2020). cargo transfer: an ISEV position paper arising from the ISEV membranes and EVs workshop. J. Extracell. Vesicles 8, 1684862 (2019).

474 11. Sutaria, D. S. et al. Low active loading of cargo into engineered extracellular 475 vesicles results in inefficient miRNA mimic delivery. J. Extracell. Vesicles 6, 1333882 (2017).

477 12. Stevanato, L., Thanabalasundaram, L., Vysokov, N. \& Sinden, J. D. Investigation of content, stoichiometry and transfer of miRNA from human neural stem cell line derived exosomes. PLoS ONE 11, 1-13 (2016). vesicle transfer by a Cre-loxP method. Nat. Protoc. 11, 87-101 (2016). 
482 14. Heath, N. et al. Endosomal escape enhancing compounds facilitate functional

483 delivery of extracellular vesicle cargo. Nanomed. 14, 2799-2814 (2019).

484 15. de Jong, O. G. et al. A CRISPR-Cas9-based reporter system for single-cell detection

485 of extracellular vesicle-mediated functional transfer of RNA. Nat. Commun. 11, $486 \quad$ (2020).

487 16. Kalluri, R. \& LeBleu, V. S. The biology, function, and biomedical applications of exosomes. Science 367, eaau6977 (2020).

489 17. Gibson, D. G. et al. Enzymatic assembly of DNA molecules up to several hundred kilobases. Nat. Methods 6, 343-345 (2009).

491 18. Hall, M. P. et al. Engineered luciferase reporter from a deep sea shrimp utilizing a novel imidazopyrazinone substrate. ACS Chem. Biol. (2012) doi:10.1021/cb3002478.

19. Votteler, J. et al. Designed proteins induce the formation of nanocage-containing extracellular vesicles. Nature 540, 292-295 (2016). are hardly delivered to target cells. bioRxiv 2020.05.20.106393 (2020) 
499 21. Somiya, M. \& Kuroda, S. Real-time luminescence assay for cytoplasmic cargo

500 delivery of extracellular vesicles. bioRxiv 2020.10.16.341974 (2020)

$501 \quad$ doi:10.1101/2020.10.16.341974.

502 22. Fredericksen, B. L. \& Whitt, M. A. Vesicular Stomatitis Virus Glycoprotein

503 Mutations That Affect Membrane Fusion Activity and Abolish Virus Infectivity. $J$

$504 \quad$ VIROL 9.

505 23. Ci, Y., Yang, Y., Xu, C. \& Shi, L. Vesicular stomatitis virus G protein

506 transmembrane region is crucial for the hemi-fusion to full fusion transition. Sci.

507 Rep. 8, 10669 (2018).

508

24. Kim, I. S. et al. Mechanism of membrane fusion induced by vesicular stomatitis virus G protein. Proc. Natl. Acad. Sci. 114, E28-E36 (2017).

25. Bowman, E. J., Siebers, a \& Altendorf, K. Bafilomycins: a class of inhibitors of membrane ATPases from microorganisms, animal cells, and plant cells. Proc. Natl. Acad. Sci. U. S. A. 85, 7972-6 (1988).

26. Yonezawa, A., Cavrois, M. \& Greene, W. C. Studies of Ebola Virus Glycoprotein-

514 Mediated Entry and Fusion by Using Pseudotyped Human Immunodeficiency Virus

515 Type 1 Virions: Involvement of Cytoskeletal Proteins and Enhancement by Tumor

$516 \quad$ Necrosis Factor Alpha. J. Virol. 79, 918-926 (2005). 
517 27. Sakata, M. et al. Analysis of VSV pseudotype virus infection mediated by rubella

$518 \quad$ virus envelope proteins. Sci. Rep. 7, 11607 (2017).

519 28. Barnea, G. et al. The genetic design of signaling cascades to record receptor

520 activation. Proc. Natl. Acad. Sci. 105, 64-69 (2008).

521 29. Zomer, A. et al. In vivo imaging reveals extracellular vesicle-mediated

522 phenocopying of metastatic behavior. Cell 161, 1046-1057 (2015).

523 30. Hung, M. E. \& Leonard, J. N. A platform for actively loading cargo RNA to

524 elucidate limiting steps in EV-mediated delivery. J. Extracell. Vesicles 1, 1-13

$525 \quad$ (2016).

526 31. Stremersch, S. et al. Comparing exosome-like vesicles with liposomes for the

527 functional cellular delivery of small RNAs. J. Controlled Release 232, 51-61

$528 \quad$ (2016).

529 32. Wang, Q. et al. ARMMs as a versatile platform for intracellular delivery of

$530 \quad$ macromolecules. Nat. Commun. 9, (2018).

531 33. Lai, C. P. et al. Visualization and tracking of tumour extracellular vesicle delivery

532 and RNA translation using multiplexed reporters. Nat. Commun. 6, 7029 (2015).

533 34. Parolini, I. et al. Microenvironmental pH Is a Key Factor for Exosome Traffic in

534 Tumor Cells. J. Biol. Chem. 284, 34211-34222 (2009). 
535 35. Wunderli-Allenspach, H. \& Ott, S. Kinetics of fusion and lipid transfer between

536 virus receptor-containing liposomes and influenza viruses as measured with the

537 octadecylrhodamine B chloride assay. Biochemistry 29, 1990-1997 (1990).

538 36. Joshi, B. S., de Beer, M. A., Giepmans, B. N. G. \& Zuhorn, I. S. Endocytosis of

539 Extracellular Vesicles and Release of Their Cargo from Endosomes. ACS Nano

$540 \quad$ acsnano.9b10033 (2020) doi:10.1021/acsnano.9b10033.

541 37. Cavrois, M., de Noronha, C. \& Greene, W. C. A sensitive and specific enzyme-

542 based assay detecting HIV-1 virion fusion in primary T lymphocytes. Nat.

543 Biotechnol. 20, 1151-1154 (2002).

544 38. Nguyen, V. V. T., Witwer, K. W., Verhaar, M. C., Strunk, D. \& Balkom, B. W. M.

545 Functional assays to assess the therapeutic potential of extracellular vesicles. $J$.

$546 \quad$ Extracell. Vesicles 10, (2020). 
A

B

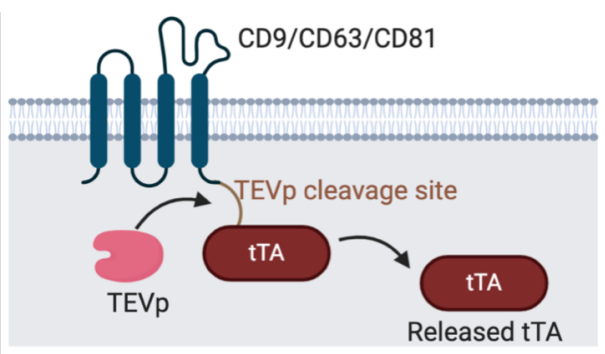

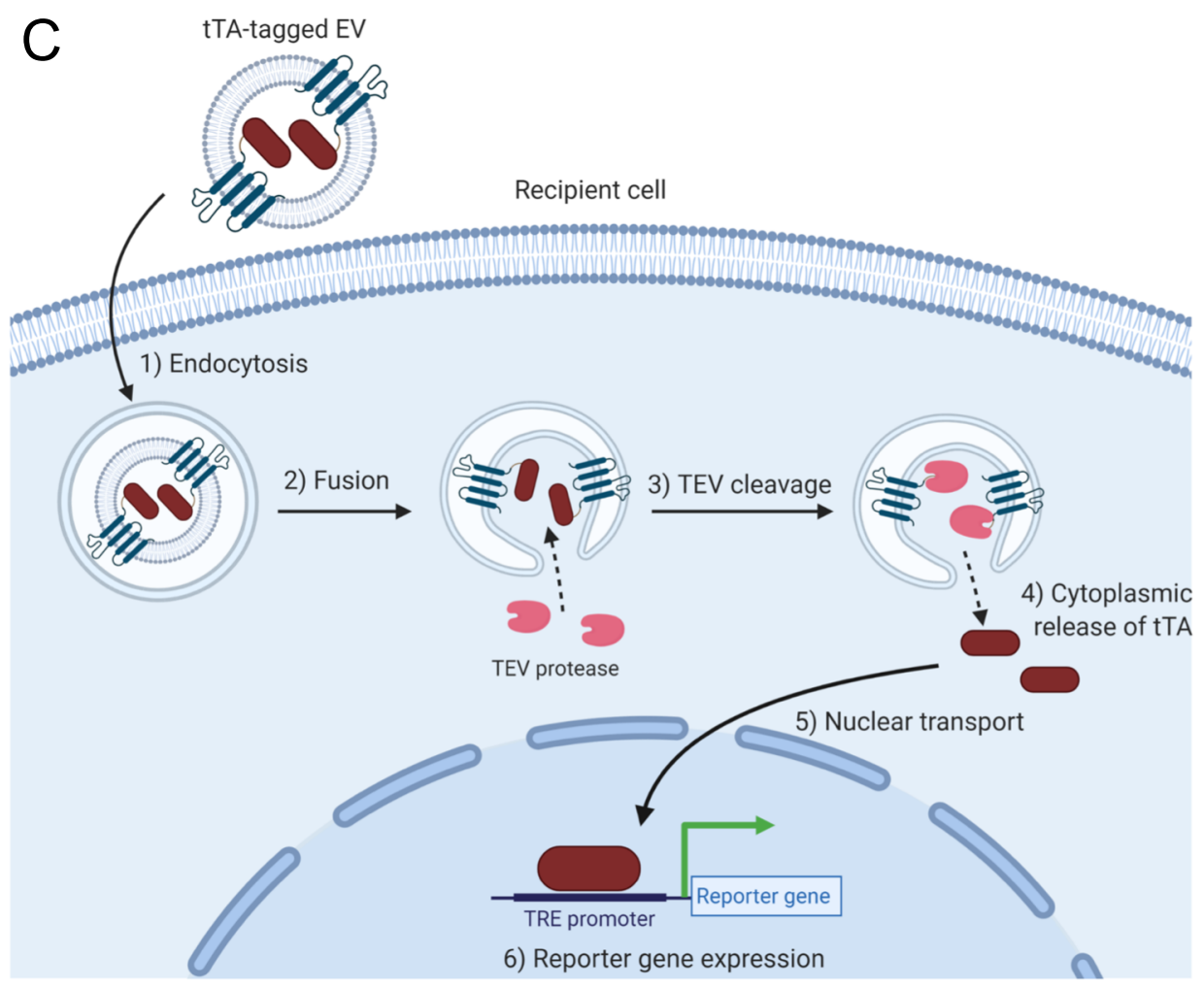


A

tTA-fused tetraspanin

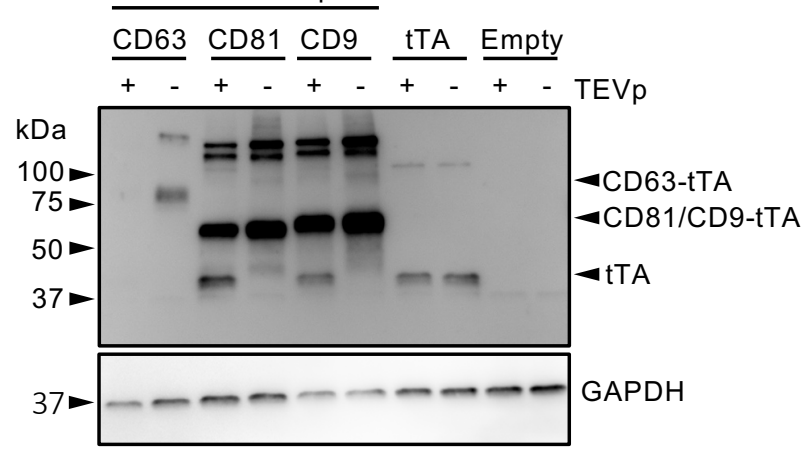

\section{B}

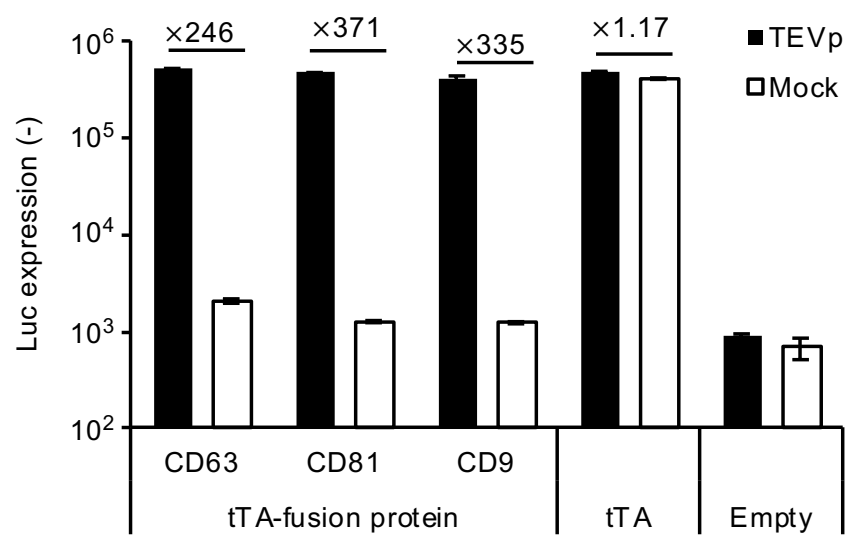



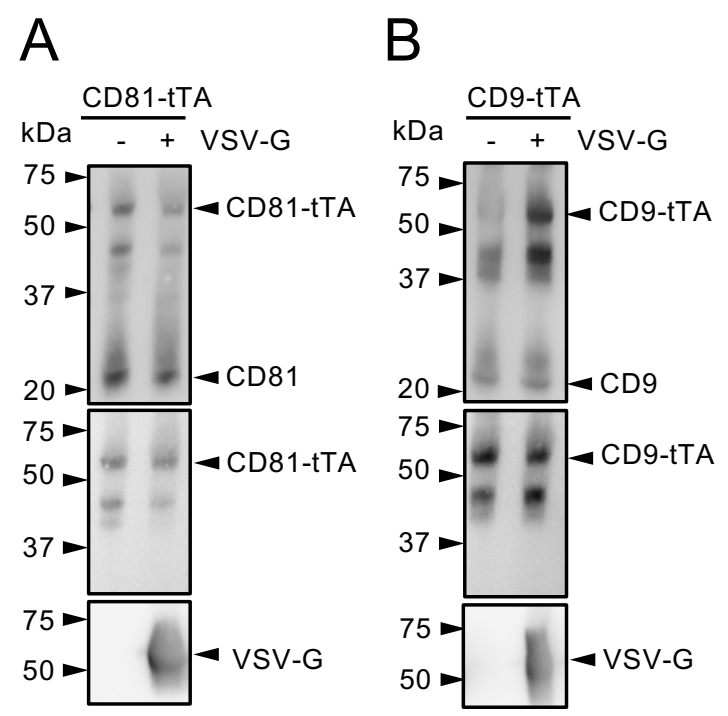

Somiya et al., Fig. 3 
A

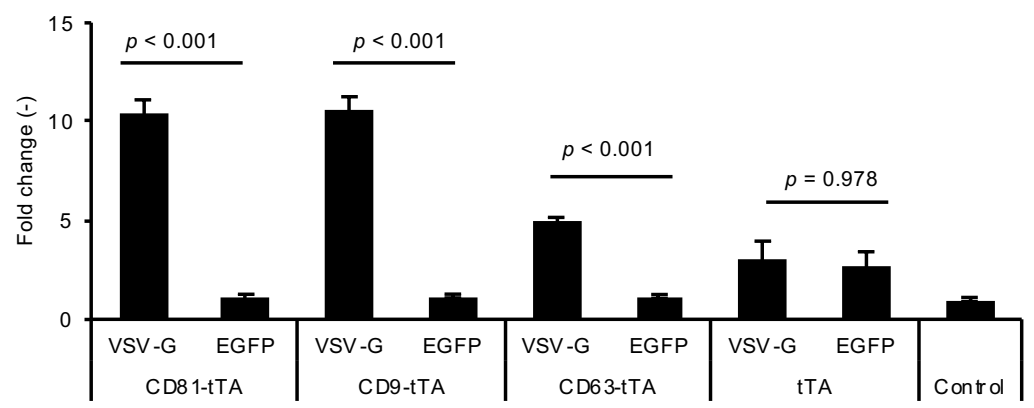

C
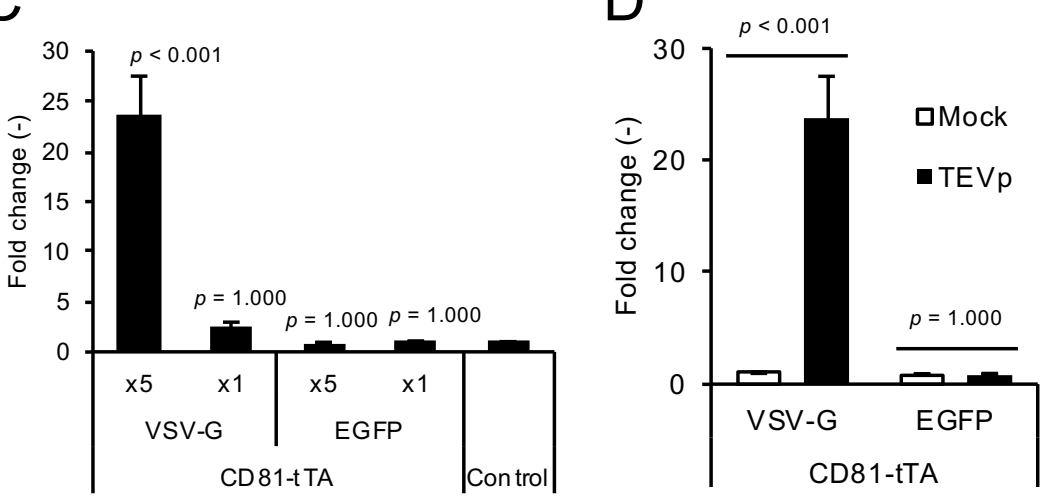

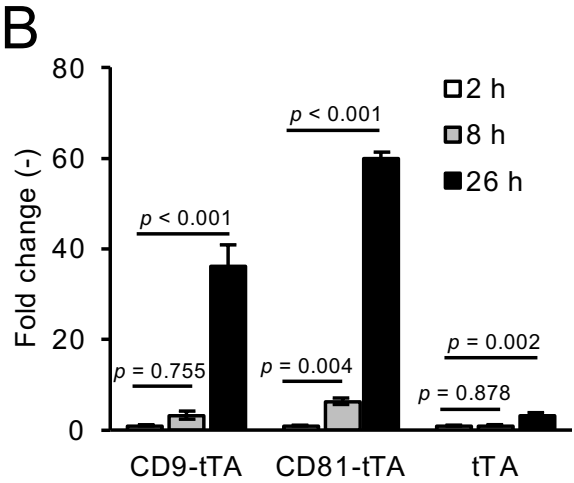

E

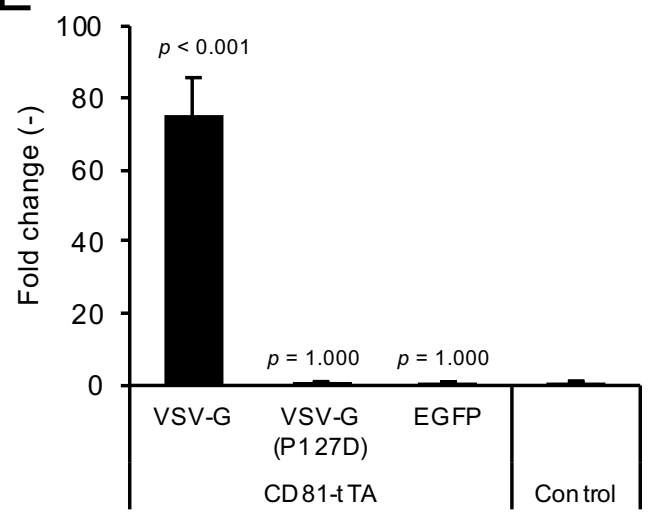

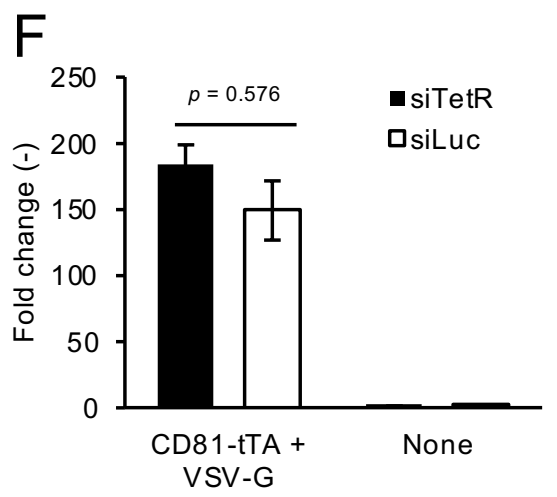

Somiya et al., Fig. 4 

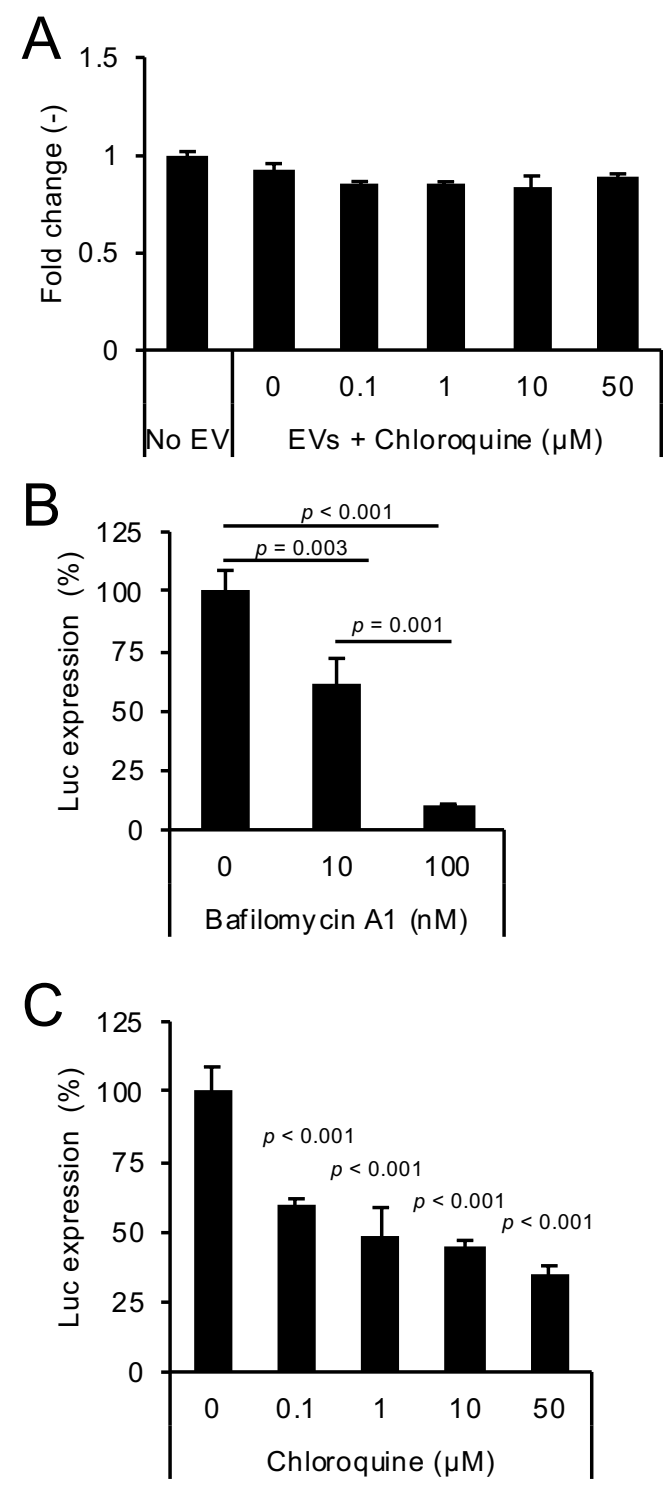

Somiya et al., Fig. 5 
A

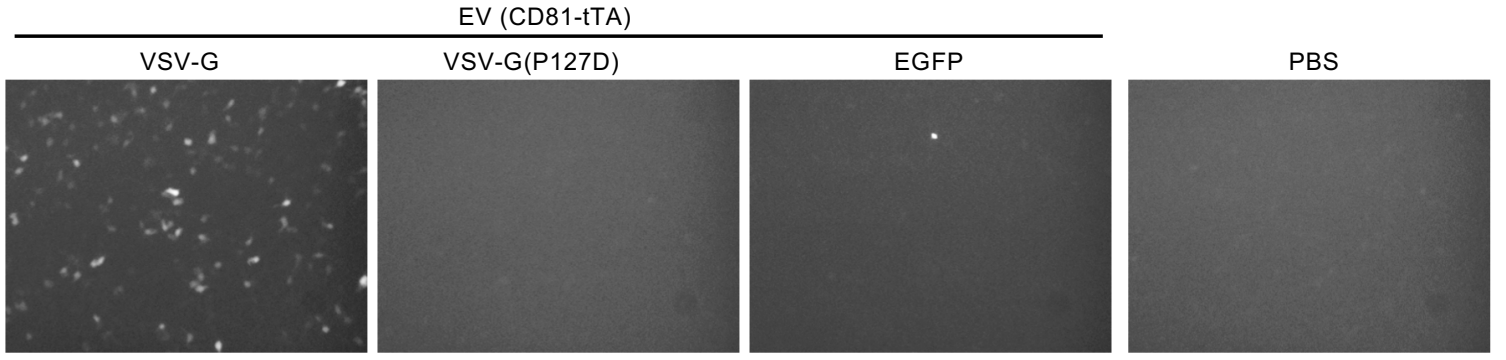

B

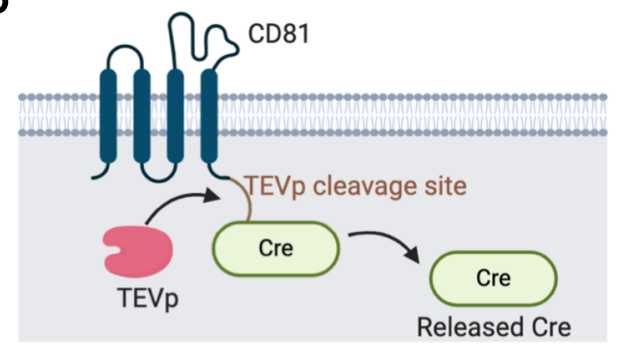

C
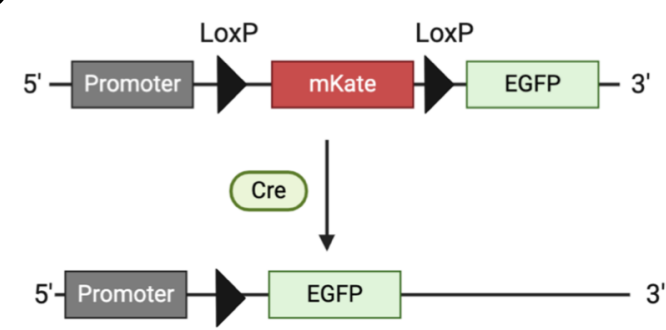

$\mathrm{D}$

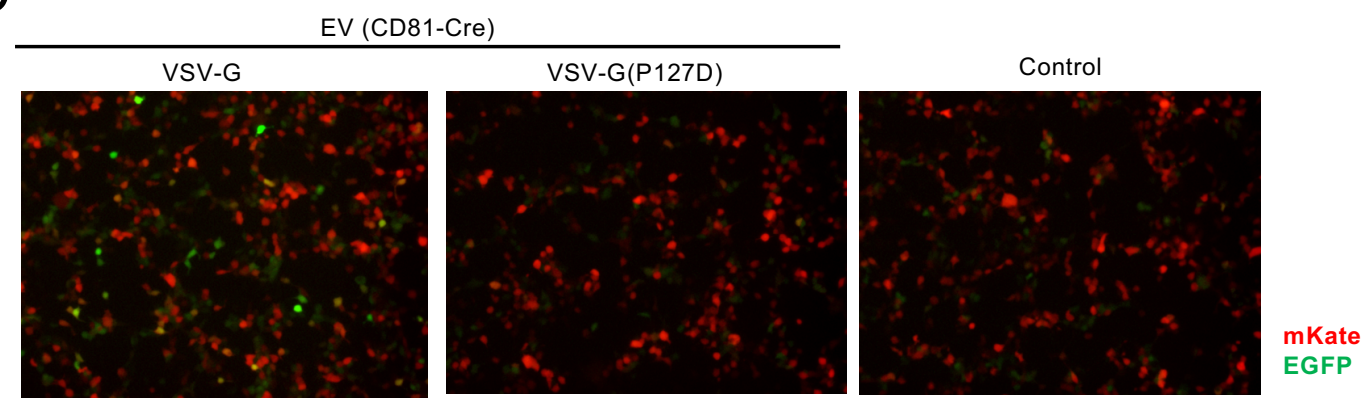

Somiya et al., Fig. 6 